\title{
Understanding changes in central nervous system function after transcatheter aortic valve replacement
}

\author{
Angelo Silverio, $\mathrm{MD}{ }^{\mathrm{a}}$ and Gennaro Galasso, $\mathrm{MD}, \mathrm{PhD}^{\mathrm{a}}$ \\ a Department of Medicine, Surgery and Dentistry, University of Salerno, Baronissi, SA, Italy
}

Received Feb 9, 2022; accepted Feb 9, 2022

doi: $10.1007 / \mathrm{s} 12350-022-02925-6$

\section{See related article, pp. 2652-2663}

Abnormalities in the cardiac sympathetic nervous (CSN) function have been documented in several heart diseases and have been directly implicated in their pathogenesis and disease progression. ${ }^{1}$ Noninvasive nuclear imaging techniques using the norepinephrine analog ${ }^{123}$ I-meta-iodobenzylguanidine (MIBG) may be employed to characterize CSN abnormalities and have demonstrated their usefulness for prognostication and risk stratification for adverse cardiac events in patients with heart failure $(\mathrm{HF}) .^{2-5}$

This assessment may be of utmost importance in HF patients with a very high risk of unfavorable outcome, such as patients with severe aortic stenosis (AS) not eligible for surgical valve replacement, who are candidates for transcatheter aortic valve replacement (TAVR). ${ }^{6,7}$ Despite the effectiveness of TAVR in normalizing the gradient across the aortic valve, about $25 \%$ of high-risk patients die from cardiovascular and noncardiovascular causes within the first year following the procedure. $^{8}$ Many Authors retain that adverse outcomes are mainly driven by pre-existing conditions at the time of TAVR procedure. ${ }^{9}$ In this setting, changes of the CNS activity and the their potential clinical effects have been poorly characterized.

The present study by Kadoya and colleagues investigated changes in MIBG imaging parameters in patients with severe AS treated with TAVR at their

Reprint requests: Gennaro Galasso, $\mathrm{MD}, \mathrm{PhD}$, Department of Medicine, Surgery and Dentistry, University of Salerno, Via Salvador Allende, 84084 Baronissi, SA, Italy; ggalasso@unisa.it

J Nucl Cardiol 2022;29:2664-6.

$1071-3581 / \$ 34.00$

Copyright (C) 2022 The Author(s) under exclusive licence to American Society of Nuclear Cardiology institution. ${ }^{10}$ This was a prospective study designed to collect information on early and late heart-mediastinum ratio $(\mathrm{H} / \mathrm{M})$ and washout rate $(\mathrm{WR})$ at baseline, within 2 weeks, and at 6-12 months after TAVR. Among 75 patients with complete imaging follow-up included in the final analysis, late $\mathrm{H} / \mathrm{M}$ significantly increased within 2 weeks, and further increased at mid-term follow-up suggesting a sustained improvement of CSN function after TAVR. Multivariable regression analysis revealed that the baseline mean aortic valve pressure gradient (mPG) was an independent predictor of late $\mathrm{H} /$ $\mathrm{M}$ increase at mid-term, and patients in the highest $\mathrm{mPG}$ value tertile $(\geq 58 \mathrm{mmHg}$ ) experienced the greatest benefit in terms of late H/M improvement, suggesting greater benefit of CSN function as the severity of AS increases.

These results were consistent with a previous study by the same group, which investigated the early effects of TAVR on MIBG parameters in patients with severe AS. ${ }^{11}$ In that analysis, the authors found a significant increase of late $\mathrm{H} / \mathrm{M}$ and a reduction of WR within 2 weeks after TAVR, and an independent association between the AS severity expressed as aortic valve area and the late $\mathrm{H} / \mathrm{M}$ improvement. The present study confirms these preliminary findings and demonstrates that the improvement in the $\mathrm{H} / \mathrm{M}$ ratio is sustained at midterm follow-up.

Beyond the limitations related to the small sample size and the inclusion of Japanese patients only, which make the results purely exploratory and not generalizable to other ethnicities, the authors should be commended for their efforts in this prospective study centered on the evaluation of a key pathophysiological pathway of HF. Late H/M reflects the number of CSN terminal endings, the noradrenaline reuptake from the synaptic cleft by the uptake-1 transporter, and the sympathetic nervous tone, and can demonstrate treatment induced changes in cardiac adrenergic activity. ${ }^{1}$ 
Several mechanisms have been proposed to explain CNS overactivation in patients with severe AS, including the suppression of the sympathoinhibitory cardiovascular reflexes (e.g., arterial baroreceptor reflex), the augmented sympathoexcitatory reflex (e.g., arterial chemoreceptor and cardiac sympathetic afferent reflexes), and the central facilitation mediated by the higher levels of angiotensin-II and cytokines or the lower production of nitric oxide, which contribute to the increased tone of sympathetic activity. ${ }^{12-14}$ The rapid release of left ventricular pressure overload and the improved hemodynamics following TAVR revert CNS impairment early and that benefit seems to be sustained at mid-term.

A significant reduction of WR at two weeks was reported by another single-center study, which investigated MIBG parameters in 31 consecutive AS patients undergoing TAVR. ${ }^{15}$ This study also showed a significant decrease in norepinephrine serum level after TAVR and that norepinephrine level before TAVI was the only independent predictor of changes in WR, suggesting that improvement of WR reflected largely the inhibition of CSN tone rather than improvement in terminal function. ${ }^{16}$

These alterations of the sympathetic cascade are thought to be early in HF pathophysiology, but may expose myocardium to toxic amounts of noradrenalin with a theoretical higher risk of arrhythmias and other life-threatening events. ${ }^{16-18}$ The study by Kadoya and colleagues suggests novel mechanisms that, together with the hemodynamic effects of TAVR, reinforce the pathophysiological basis of the beneficial effects of TAVR, particularly in patients with more severe highgradient forms of AS.

Nevertheless, it is still unknown whether changes in MIBG imaging parameters correspond to an outcome benefit in the individual patient. In their manuscript, the authors hypothesized several potential clinical implications but, to date, we do not have sufficient evidence to implement this information in TAVR candidacy or patient management after procedure.

In a previous study by the same group, 108 AS patients were evaluated with MIBG imaging before and soon after TAVR and followed to evaluate the occurrence of major adverse cardiac events (MACE), defined as a composite of all-cause death, non-fatal myocardial infarction, and HF hospitalization. ${ }^{19}$ After adjustment for possible confounders, the improvement of late $\mathrm{H} / \mathrm{M}$ was significantly associated with MACE at a median 1year follow-up (adjusted HR: 0.233; 95\% CI 0.0640.856), suggesting some usefulness for prognostic stratification in patients candidates for TAVR. Conversely, in the present study focused on mid-term changes of MIBG imaging, the authors did not provide outcome information nor investigate the prognostic implication of CNS re-assessment following TAVR, which prevents a comprehensive appraisal of the clinical perspectives of these results.

It should be emphasized that the final study population included only 75 of the 183 AS patients who underwent TAVR during the recruitment period. Seven patients died after discharge and 21 required additional medical treatments, which was an exclusion criterion for subsequent MIBG evaluation in order to avoid confounding. Therefore, the improvement in MIBG parameters might be restricted to less severe patients and might not reflect CNS changes in the general AS population undergoing TAVR.

Eventually, we cannot overlook that most of the evidence on this topic comes from studies conducted by one group on the same cohort of patient. External studies are advisable to confirm the validity of this preliminary experience, and to evaluate the possible implications for patient prognostic stratification and TAVR treatment monitoring in a wider multicenter population.

There is still a long way to go before understanding if, when and which patients with severe AS should be evaluated with MIBG imaging in view of TAVR treatment, as well as the cost-effectiveness to do so.

\section{Disclosures}

No conflict of interest or any financial support to declare.

\section{References}

1. Carrió I, Cowie MR, Yamazaki J, Udelson J, Camici PG. Cardiac sympathetic imaging with mIBG in heart failure. JACC Cardiovasc Imaging 2010;3:92-100.

2. Jacobson AF, Senior R, Cerqueira MD, Wong ND, Thomas GS, Lopez VA. Myocardial iodine-123 meta-iodobenzylguanidine imaging and cardiac events in heart failure. Results of the prospective ADMIRE-HF (AdreView Myocardial Imaging for Risk Evaluation in Heart Failure) study. J Am Coll Cardiol 2010;55:2212-21.

3. Narula J, Gerson M, Thomas GS, Cerqueira MD, Jacobson AF. 123I-MIBG imaging for prediction of mortality and potentially fatal events in heart failure: The ADMIRE-HFX study. J Nucl Med 2015;56:1011-8.

4. Wakabayashi T, Nakata T, Hashimoto A, Yuda S, Tsuchihashi K, Travin MI, et al. Assessment of underlying etiology and cardiac sympathetic innervation to identify patients at high risk of cardiac death. J Nucl Med 2001;42:1757-67.

5. Paolillo S, Rengo G, Pagano G, Pellegrino T, Savarese G, Femminella GD, et al. Impact of diabetes on cardiac sympathetic innervation in patients with heart failure: A 123I meta-iodobenzylguanidine (123I MIBG) scintigraphic study. Diabetes Care 2013;36:2395-401.

6. Kapadia SR, Tuzcu EM, Makkar RR, Svensson LG, Agarwal S, Kodali S, et al. Long-term outcomes of inoperable patients with 
aortic stenosis randomly assigned to transcatheter aortic valve replacement or standard therapy. Circulation 2014;130:1483-92.

7. Sedaghat A, Al-Rashid F, Sinning JM, Wendt D, Thielmann M, Grube E, et al. Outcome in TAVI patients with symptomatic aortic stenosis not fulfilling PARTNER study inclusion criteria. Catheter Cardiovasc Interv 2015;86:1097-104.

8. Gilard M, Eltchaninoff H, Iung B, Donzeau-Gouge P, Chevreul K, Fajadet J, et al. Registry of transcatheter aortic-valve implantation in high-risk patients. N Engl J Med 2012;366:1705-15.

9. Lantelme P, Eltchaninoff H, Rabilloud M, Souteyrand G, Dupré M, Spaziano M, et al. Development of a risk score based on aortic calcification to predict 1-year mortality after transcatheter aortic valve replacement. JACC Cardiovasc Imaging 2019;12:123-32.

10. Kadoya Y, Zen K, Tamaki N, Nakamura S, Fujimoto T, Yashige $\mathrm{M}$, et al. Serial changes in cardiac sympathetic nervous function after transcatheter aortic valve replacement: A prospective observational study using (123)I-meta-iodobenzylguanidine imaging. J Nucl Cardiol 2021. https://doi.org/10.1007/s12350-021-02799-0.

11. Kadoya Y, Zen K, Tamaki N, Ito N, Kuwabara K, Yamano M, et al. Early effects of transcatheter aortic valve replacement on cardiac sympathetic nervous function assessed by (123)Imetaiodobenzylguanidine scintigraphy in patients with severe aortic valve stenosis. Eur J Nucl Med Mol Imaging 2020;47:165767.

12. Watson AM, Hood SG, May CN. Mechanisms of sympathetic activation in heart failure. Clin Exp Pharmacol Physiol 2006;33:1269-74.

13. Li YF, Wang W, Mayhan WG, Patel KP. Angiotensin-mediated increase in renal sympathetic nerve discharge within the PVN:
Role of nitric oxide. Am J Physiol Regul Integr Comp Physiol 2006;290:R1035-43.

14. Garrido AM, Griendling KK. NADPH oxidases and angiotensin II receptor signaling. Mol Cell Endocrinol 2009;302:148-58.

15. Sobajima M, Ueno H, Onoda H, Kuwahara H, Tanaka S, Ushijima $\mathrm{R}$, et al. Transcatheter aortic valve implantation improves cardiac sympathetic nerve activity on (123)I-metaiodobenzylguanidine myocardial scintigraphy in severe aortic valve stenosis. Circ J 2018;82:579-85.

16. Zelt JGE, deKemp RA, Rotstein BH, Nair GM, Narula J, Ahmadi A, et al. Nuclear imaging of the cardiac sympathetic nervous system: A disease-specific interpretation in heart failure. JACC Cardiovasc Imaging 2020;13:1036-54.

17. Haider N, Baliga RR, Chandrashekhar Y, Narula J. Adrenergic excess, hNET1 down-regulation, and compromised mIBG uptake in heart failure poverty in the presence of plenty. JACC Cardiovasc Imaging 2010;3:71-5.

18. Silverio A, Polito MV, Pace L, D'Auria F, Vitulano G, Scarano M, et al. Predictors of outcome in patients with de novo diagnosis of heart failure with reduced ejection fraction: Role of combined myocardial and lung Iodine-123 Meta-Iodobenzylguanidine imaging. J Nucl Cardiol 2021;28:72-85.

19. Kadoya Y, Zen K, Tamaki N, Yashige M, Takamatsu K, Ito N, et al. Prognostic value of cardiac (123) I-metaiodobenzylguanidine imaging for predicting cardiac events after transcatheter aortic valve replacement. ESC Heart Fail 2021;8:1106-16.

Publisher's Note Springer Nature remains neutral with regard to jurisdictional claims in published maps and institutional affiliations. 